\title{
INDICE DE PULSATILIDAD PROMEDIO DEL DOPPLER DE LAS ARTERIAS UTERINAS COMO PREDICTOR DE MACROSOMIA FETAL. AÑOS 2011-2012
}

\author{
Oscar Antonio Limay Ríos¹, Elías Alexis Valladares Gutiérrez², Hugo Camarena Estela², \\ Amadeo Sánchez Góngora ${ }^{3}$, Erasmo Huertas Tacchino ${ }^{4}$, Walter Castillo Urquiaga ${ }^{1}$, Mario Zárate Girao1.
}

\begin{abstract}
RESUMEN
Objetivo. Determinar la utilidad del índice de pulsatilidad (IP) promedio del doppler de las arterias uterinas antes de las 30 semanas de gestación como predictor de macrosomía en recién nacidos atendidos en el Instituto Nacional Materno Perinatal durante el periodo 2011 - 2012. Materiales y métodos. Estudio retrospectivo, transversal y analítico. Se determinó el índice de pulsatilidad promedio de las arterias uterinas a 232 gestantes menores de 30 semanas y se calculó el peso neonatal al nacimiento en función a su edad gestacional para obtener dos grupos; el primero con neonatos con adecuado peso al nacer y el segundo con neonatos macrosómicos $(n=18 / 7,8 \%)$, los cuales fueron comparados utilizando desviaciones estándar, correlación de Pearson, T de Student y la curva Receptor-operador (ROC). Resultados. Se encontró variación entre ambos grupos en los cuatro índices promedio (pico sistólico, pico diastólico, índice de pulsatilidad e índice de resistencia) existiendo correlación con la prueba de Pearson. EI IP promedio fue significativamente menor $(p=0,001)$ en los neonatos macrosómicos que en aquellos sin macrosomía, observándose una relación inversamente proporcional entre los valores del IP con la edad gestacional. Para la curva ROC se establecieron arbitrariamente dos puntos de corte tentativos para el IP promedio de las arterias uterinas, el primer punto $\leq 0,65$ se presentó en el $22,2 \%$ de los neonatos macrosómicos y en el $10,3 \%$ de los neonatos sin macrosomía; y el segundo punto de corte $\leq 0,75$ incluyó al $50 \%$ de los neonatos con macrosomía y al $20,6 \%$ de aquellos sin macrosomía. Conclusión. Un valor de la media del índice de pulsatilidad de las arterias uterinas $\leq 0,75$ se asoció con macrosomía al nacer.
\end{abstract}

Palabras clave: Doppler; Arterias uterinas; Macrosomía; Indice de pulsatilidad; Predicción (fuente: DeCS BIREME).

\section{AVERAGE PULSATILITY INDEX OF UTERINE ARTERY DOPPLER AS A PREDICTOR OF FETAL MACROSOMIA. YEARS 2011-2012}

\begin{abstract}
Objective. To determine the usefulness of the mean pulsatility index (PI) of uterine artery doppler before 30 gestation weeks as a predictor of macrosomia in newborns treated at the National Perinatal Maternal Institute during the period 2011- 2012. Material and methods. A retrospective, cross-sectional and analytical study. It was determined the mean pulsatility index of the uterine arteries to 232 pregnant women under 30 gestation weeks' and birth weight was calculated according to birth gestational age for two groups, the first with infants with appropriate birth weight and the second with macrosomic neonates $(n=18 / 7,8 \%)$, which were compared using standard deviations, Pearson correlation, Student $t$ test and receiver-operator curve (ROC). Results. Variation was found between the two groups in the four mean indices (peak systolic, peak diastolic, pulsatility index and resistance index), existing correlation with the Pearson test. The mean IP was significantly lower ( $p=$ $0,001)$ in macrosomic infants than in those without macrosomia, showing an inverse relationship between IP values with gestational age. For ROC curve were arbitrarily set two tentative breakpoints for the mean IP of the uterine arteries, the first point $\leq 0,65$ was presented in $22,2 \%$ of macrosomic infants and $10,3 \%$ of the infants without macrosomia, and the second cutoff $\leq 0,75$ included $50 \%$ of infants with macrosomia and $20,6 \%$ of those without macrosomia, Conclusion. A value of mean pulsatility index of the uterine arteries $\leq 0,75$ was associated with macrosomia at birth.
\end{abstract}

Key words: Doppler uterine arteries; Macrosomia; Pulsatility index; Prediction (source: MeSH NLM).

\section{INTRODUCCIÓN}

El crecimiento fetal humano, es un proceso en el cual aumenta la masa celular del feto, mediante el incremento del número y tamaño de sus células, así como de la matriz intercelular ${ }^{1-4}$. Una serie de procesos que suceden simultáneamente durante el crecimiento del feto, como la diferenciación orgánica, el aumento de peso y el desarrollo de la función corporal, lo prepara para vivir fuera del útero en condiciones óptimas. Los procesos celulares característicos del crecimiento fetal (aumento de masa, síntesis de proteínas y diferenciación) no son bien

Médico GinecoObstetra Asistente de la Unidad de Medicina Fetal. Instituto Nacional Materno Perinatal. Lima-Perú.

2 Médico GínecoObstetra Asistente del Servicio de Emergencia. Instituto Nacional Materno Perinatal. Lima-Perú. Magíster en Docencia e Investigación en Salud.

Médico Obstetra. Director Ejecutivo de Investigación Docencia y Atención en Obstetricia y Ginecología.

4 Jefe de la Unidad de Medicina Fetal. Instituto Nacional Materno Perinatal. Profesor de la Facultad de Medicina - Universidad Nacional Mayor de San Marcos. Lima-Perú. 
conocidos $^{2,5}$. Sin embargo, el genotipo fetal contribuye con casi $15 \%$ de las variaciones en el peso al nacer debido a ciertas tendencias heredadas como género, características raciales-étnicas y contribuciones genéticas maternas y paternas ${ }^{6}$.

La adecuada evaluación intrauterina del crecimiento fetal es de gran importancia en el manejo y toma de decisiones en la práctica obstétrica actual, con el fin de prevenir complicaciones y mejorar el pronóstico fetal, pues tanto el bajo peso al nacer como la macrosomía fetal se asocian con un mayor morbi-mortalidad maternoperinatal $^{5-10}$.

La definición clásica de un recién nacido grande para la edad gestacional es de aquel con un peso mayor o igual al percentil 90 para una edad gestacional dada 5,27 .

En los últimos años, la incidencia de macrosomía ha aumentado considerablemente, reportándose tasas que oscilan entre 10 a $13 \%$ a nivel mundial, cuando se utiliza como valor neto un peso de nacimiento superior a 4000 gramos $^{12-19}$. En el Perú, llama la atención que una población de nivel socioeconómico medio bajo y bajo tuviera una prevalencia nacional de macrosomía fetal de $11.37 \%$, que aumenta a $14.34 \%$ en Lima ${ }^{12,20}$.

La capacidad de estudiar el flujo sanguíneo del feto y la circulación placentaria en forma no invasiva, mediante velocimetría doppler, constituye el área de mayor avance en medicina perinatal durante los últimos años ${ }^{12-21}$.

El desarrollo adecuado de la circulación útero placentaria es de importancia fundamental en el logro de un embarazo normal ${ }^{22}$. Aproximadamente el $80 \%$ de la irrigación uterina durante el embarazo proviene de ambas arterias uterinas, que se originan de la división anterior de la arteria ilíaca interna ${ }^{23}$. Durante la invasión del trofoblasto, primero a nivel decidual, y luego a nivel miometrial, las arterias espirales cambian su estructura musculo-elástica por tejido fibrinoide, disminuyendo notoriamente la resistencia global uterina. Como resultado del aumento del débito cardíaco materno, se produce un aumento sustancial del flujo uterino a través del embarazo, desde $50 \mathrm{ml} / \mathrm{min}$ en primer trimestre a 500 $\mathrm{ml} / \mathrm{min}$ hacia el término ${ }^{24}$.

En condiciones normales, el flujo de ambas arterias umbilicales es comparable y aumenta progresivamente a través del embarazo, representando el $40 \%$ del débito cardíaco combinado fetal ${ }^{7}$. El lecho vascular placentario no es inervado y, por lo tanto, es refractario a sustancias vasoconstrictoras circulantes, por lo que los mecanismos regulatorios locales adquieren mayor importancia. La circulación útero placentaria y umbilico-placentaria son circuitos de alto flujo y baja resistencia; característica que hace que sus vasos posean ondas de velocidades de flujo doppler absolutamente reconocibles y específicas, compartiendoel flujo uterino total entre la placenta y el miometrio ${ }^{25,26}$.
En situaciones de hipoxia existen cambios hemodinámicos e histológicos placentarios, como: vascularización anormal de las vellosidades terciarias, áreas de isquemia, infarto, y aterosis; con el consiguiente aumento de los índices umbilicoplacentarios que con la hipoxia asociada explican una serie de ajustes cardiovasculares fetales. Durante la hipoxia fetal se produce una redistribución del flujo sanguíneo en favor de cerebro, miocardio y glándulas suprarrenales, a expensas de territorio pulmonar, renal, esplácnico, muscular y piel $^{27,28}$. Paralelamente, existe un privilegio de circuitos intrafetales (extra e intracardíacos) de mayor velocidad y oxigenación hacia el cerebro. Una adecuada interpretación clínica de cada uno de estos fenómenos constituye la base de la introducción de la velocimetría Doppler en la práctica obstétrica ${ }^{29}$.

Numerosos estudios ${ }^{21,22-26}$ han evaluado la validez de la velocimetría doppler para predecir complicaciones en la placentación. Así, una alternativa para la predicción de macrosomía fetal es la evaluación doppler de las arterias uterinas, ya que está demostrado que hay una relación directa entre el aporte sanguíneo de la madre al feto y la ganancia de peso fetal.

Por esta razón, con el fin de contribuir en la prevención y disminución de las tasas de morbi-mortalidad materna, fetal y neonatal se realizó el presente estudio con el objetivo de determinar la utilidad el índice de pulsatilidad (IP) promedio del doppler de las arterias uterinas antes de las 30 semanas de gestación como predictor de macrosomía en recién nacidos atendidos en el Instituto Nacional Materno Perinatal durante el periodo 2011 - 2012.

\section{MATERIALES Y MÉTODOS}

Estudio retrospectivo, transversal y analítico. Se calculó el índice de pulsatilidad promedio de las arterias uterinas mediante ultrasonografía doppler vía abdominal o transvaginal a 232 gestantes menores de 30 semanas que acudieron a la Unidad de Medicina Fetal del Instituto Nacional Materno Perinatal (INMP) desde abril del 2011 hasta el 01 de julio del 2012. Se determinó el peso al nacimiento en función a su edad gestacional y se obtuvo dos grupos, el primero con neonatos con peso adecuado al nacer y el segundo con neonatos macrosómicos. El tipo de muestreo fue probabilístico y la técnica de muestreo fue simple aleatorio.

Se incluyó a todas las gestante con feto único cuya edad gestacional se encontraba entre las 12 a 30 semanas, tenían 3 controles prenatales como mínimo, feto vivo sin malformaciones y se controlaban el embarazo hasta la fecha del parto en la institución. Se excluyó a aquellas gestantes con más de 30 semanas al momento de la captación, sin controles prenatales, óbito fetal o con malformaciones fetales y que no se hayan atendido en el INMP o cuyo seguimiento haya sido de difícil acceso.

En la ejecución del presente trabajo se estableció dos grupos de gestantes, el primero (grupo de estudio) con índice de pulsatilidad promedio de las uterinas $\leq 0.75 \mathrm{y}$ el 
segundo (grupo comparativo) a gestantes con Índice de pulsatilidad promedio de las uterinas $\leq 0,65$.

Para la evolución ultrasonográfica se verificó que el transductor haya sido ubicado en forma para-sagital a nivel de la unión cervico-ítsmica, 2-3 cm medial a la espina ilíaca antero superior. En esta posición se identificó ambas arterias uterinas a nivel de su nacimiento desde la división anterior de las respectivas arterias ilíacas internas, para lo cual se ubicó a la paciente en decúbito dorsal y se dispuso el transductor de ultrasonido doppler color en la pared uterina lateral baja, buscando medialmente la zona correspondiente.

Los datos se registraron en una base elaborada en la hoja de cálculo del programa IBM Statistics SPSS 19 tomando en cuenta todas las variables e indicadores, realizando el análisis descriptivo y analítico con el mismo paquete estadístico. Se consideró el peso al nacer mayor del percentil 90 de acuerdo a la edad gestacional según la tabla de Ticona \& Huanca ${ }^{11}$.

Se utilizó el IP ${ }^{30}$ por que las evidencias indican que es el mejor índice para la valoración doppler obstétrica, tiene menos variación y es la menos afectada por el ángulo de insonación, incluso cuando la ausencia de flujo llega a la línea de base.

Las variables cuantitativas se determinaron mediante medidas de tendencia central (mediana) y medidas de dispersión (desviación estándar) y para las variables cualitativas se determinó frecuencias y porcentajes. Se plantea ver el grado de asociación de las variables estudiadas sobre la base del cálculo de la prueba Chi-cuadrado. Se procedió a utilizar el estadístico Coeficiente de regresión múltiple $\left(\mathbf{r}^{2}\right)$ con un nivel de significación estadística alfa $<0,05$. Las variables continuas fueron analizadas con la Prueba t de Student.

\section{RESULTADOS}

Se estudió a 232 gestantes menores de 30 semanas y se calculó el peso neonatal al nacimiento en función a su

Tabla 1. Distribución de los resultados perinatales en la población estudiada. Unidad de Medicina Fetal - INMP. Años 2011-2012.

\begin{tabular}{lrr}
\hline \multicolumn{1}{c}{ Resultados perinatales } & $\mathbf{n}$ & \multicolumn{1}{c}{$\%$} \\
\hline Macrosomía & & \\
$\mathrm{Si}$ & 18 & $7,8 \%$ \\
$\mathrm{No}$ & 214 & $92,2 \%$ \\
$\mathrm{RCIU}$ & & \\
$\mathrm{Si}$ & 29 & $12,5 \%$ \\
$\mathrm{No}$ & 203 & $87,5 \%$ \\
Resultado perinatal adverso & & \\
$\mathrm{Si}$ & 47 & $21 \%$ \\
$\mathrm{No}$ & 183 & $78,9 \%$ \\
Total & $\mathbf{2 3 2}$ & $\mathbf{1 0 0 \%}$ \\
\hline
\end{tabular}

Tabla 2. Comparación de las medias de PS, PD, IP e IR de la ecografía doppler de las arterias uterinas antes de las 30 semanas. Unidad de Medicina Fetal - INMP. Años 2011-2012.

\begin{tabular}{ccc}
\hline \multirow{2}{*}{ Parámetro } & \multicolumn{2}{c}{ Macrosomía } \\
\cline { 2 - 3 } & Si & No \\
\cline { 2 - 3 } & Media \pm DS & Media \pm DS \\
\hline PS Promedio & $129,3 \pm 60,1$ & $100,6 \pm 29,4$ \\
PD Promedio & $64,0 \pm 42,9$ & $43,2 \pm 19,5$ \\
IP Promedio & $0,8 \pm 0,3$ & $1,1 \pm 0,4$ \\
IR Promedio & $0,5 \pm 0,1$ & $0,6 \pm 0,1$ \\
\hline
\end{tabular}

edad gestacional para obtener dos grupos; el primero con neonatos con adecuado peso al nacer y el segundo con neonatos macrosómicos ( $n=18 / 7,8 \%)$ atendidas en la Unidad de Medicina Fetal del Instituto Nacional Materno Perinatal entre los años 2011 a 2012 de acuerdo con los criterios de inclusión y exclusión.

La tabla 1 resume los resultados perinatales. De las 232 gestantes a quienes se les realizó ecografía doppler de las arterias uterinas antes de las 30 semanas, 7 (21\%) tuvieron resultados perinatales adversos. De este grupo, el 7,8\% tuvo macrosomía fetal.

El pico sistólico promedio en los neonatos macrosómicos fue de 129,3 \pm 60 , 1 superior comparado con el pico sistólico promedio de los no macrosómicos $100,6 \pm 29,4$. El pico diastólico promedio en los neonatos macrosómicos fue de $64,0 \pm 42,9$ superior comparado con el pico diastólico promedio de los no macrosómicos $43,2 \pm 219,5$. El índice de pulsatilidad promedio en los neonatos macrosómicos fue de 0,8 \pm 0,3 inferior comparado con el índice de pulsatilidad promedio de los no macrosómicos 1,1 \pm 0,4 . El índice de resistencia promedio en los neonatos macrosómicos fue de 0,8 \pm 0,3 inferior comparado con el índice de resistencia promedio de los no macrosómicos $0,6 \pm 0,1$ (Tabla 2).

Existió correlación $(r=0,222)$ entre la media del pico sistólico y la presencia de macrosomía al nacer $(p=0,001)$. Existió mayor correlación $(r=0,245)$ entre el pico diastólico promedio y la presencia de macrosomía $(p<0,001)$.

Tabla 3. Matriz de correlación de Pearson para la media del PS, PD, IP, IR de las arterias uterinas antes de las 30 semanas. Unidad de Medicina Fetal - INMP. Años 2011-2012.

\begin{tabular}{cccccc}
\hline & Macrosomía & $\begin{array}{c}\text { PS } \\
\text { Media }\end{array}$ & $\begin{array}{c}\text { PD } \\
\text { Media }\end{array}$ & $\begin{array}{c}\text { IP } \\
\text { Media }\end{array}$ & $\begin{array}{c}\text { IR } \\
\text { Media }\end{array}$ \\
\hline $\begin{array}{c}\text { Correlación } \\
\text { de Pearson } \\
\alpha\end{array}$ & 1 & $-.222^{* *}$ & $-.245^{* *}$ & $.161^{*}$ & $.167^{*}$ \\
$\mathrm{~N}$ & 232 & .001 & .000 & .014 & .011 \\
\hline
\end{tabular}

** La correlación es significativa al nivel 0,01 (bilateral).

* La correlación es significante al nivel 0,05 (bilateral). 
Tabla 4. Prueba $T$ para la media del PS, PD, IP, IR de las arterias uterinas antes de las 30 semanas. Unidad de Medicina Fetal - INMP. Años 2011-2012.

\begin{tabular}{cccc}
\hline \multirow{2}{*}{ Macrosomía } & SI & NO & \multirow{2}{*}{$\mathbf{p}$} \\
\cline { 2 - 3 } & $\mathbf{X} \pm$ DS & $\mathbf{X} \pm \mathbf{D S}$ & \\
\hline PS Promedio & $129,32 \pm 69,06$ & $100,60 \pm 29,36$ & 0,097 \\
PD Promedio & $64,01 \pm 42,84$ & $43,19 \pm 19,51$ & 0,056 \\
IP Promedio & $0,82 \pm 0,256$ & $10,80 \pm 0,45$ & 0,001 \\
IR Promedio & $0,51 \pm 0,09$ & $0,58 \pm 0,12$ & 0,011 \\
\hline
\end{tabular}

Hubo correlación $(r=0,161)$ entre la media del Índice de pulsatilidad y la presencia de macrosomía neonatal $(p=0,014)$. Existió correlación $(r=0,167)$ entre la media del Índice de resistencia y la presencia de macrosomía al nacer ( $p=0,011)$ (Tabla 3).

En la prueba $\mathrm{T}$ para grupos analizando los cuatro índices estudiados, el único índice que evidenció una diferencia significativa fue el índice de pulsatilidad promedio $(p=0,001)$ donde se aprecia que la media del pico sistólico de los neonatos macrosómicos es significativamente menor que los no macrosómicos. Con respecto al índice de resistencia se apreció lo mismo, aunque sin significancia estadística; mientras que los picos sistólicos y diastólicos definitivamente no mostraron diferencia (Tabla 4).

La figura 1 muestra la media del índice de pulsatilidad de las arterias uterinas de los neonatos macrosómicos tienen una tendencia hacia los valores bajos.

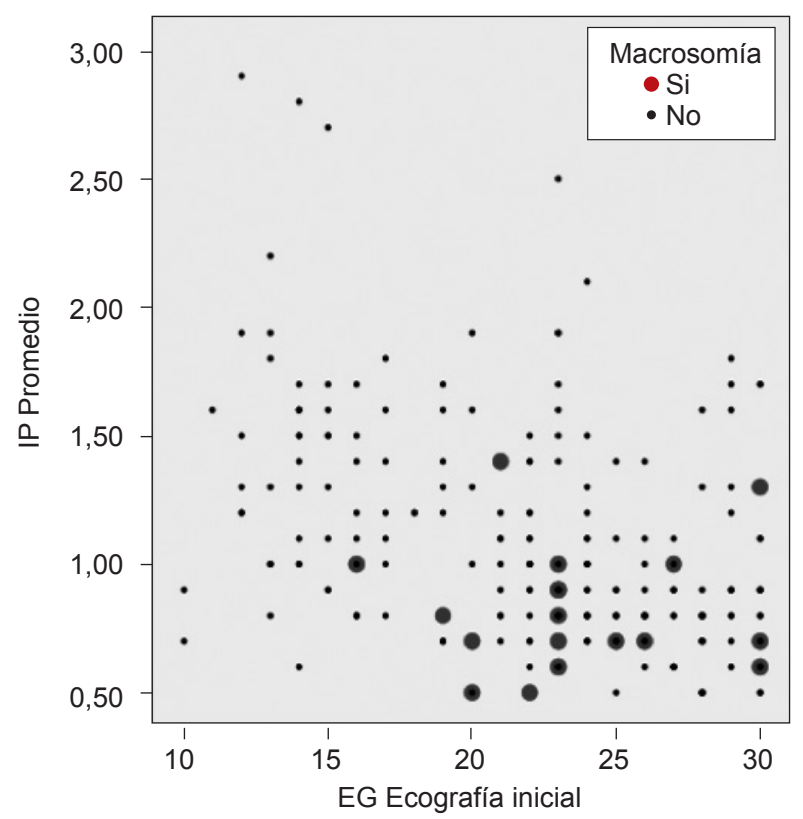

Figura 1. Distribución de los neonatos relacionado la presencia de macrosomía con los valores del índice de pulsatilidad de las arterias uterinas antes de las 30 semanas. Unidad de Medicina Fetal - INMP. Años 2011-2012.

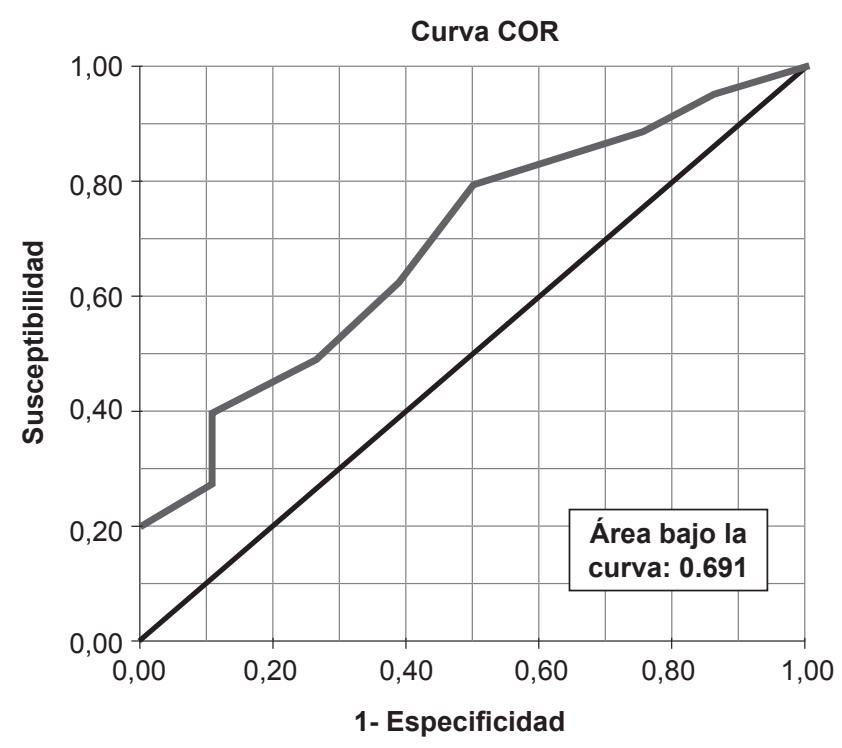

Los segmentos diagonales son producidos por los empates.

Figura 2. Curva ROC para la predicción de macrosomía fetal según los valores de la media del índice de pulsatilidad de las arterias uterinas. Unidad de Medicina Fetal - INMP. Años 20112012.

El área bajo la curva ROC que detecta los casos de macrosomía fetal correspondió a una media de IP de las arterias uterinas de 0,691, lo que significa que el punto de corte ideal se encuentra alrededor de este valor de IP, por lo cual se estableció dos puntos de corte tentativos para ver con cual el mejor (IP $\leq 0,65$ e IP $\leq$ $0,75)$. Ver figura 2.

La tabla 5 muestra la comparación de los puntos de corte establecidos. Cuando el punto del índice de Pulsatilidad fue $\leq 0,65$ y se presentó en el $22,2 \%$ de los fetos macrosómicos y en el $10,3 \%$ de los fetos sin macrosomía. Con un índice de Pulsatilidad como punto de corte $\leq 0,75$ se incluyó al $50 \%$ de los fetos con macrosomía, así como al $20,6 \%$ de los fetos sin macrosomía; lo cual representó la mejor capacidad predictiva.

Tabla 5. Comparación de los posibles puntos de corte de la media del índice de pulsatibilidad de las arterias uterinas. Unidad de Medicina Fetal - INMP. Años 20112012.

\begin{tabular}{ccccc}
\hline \multirow{2}{*}{$\begin{array}{l}\text { Puntos de } \\
\text { corte posibles }\end{array}$} & \multicolumn{5}{c}{ Macrosomía } \\
\cline { 2 - 5 } & \multicolumn{2}{c}{ Si } & \multicolumn{3}{c}{ No } \\
\cline { 2 - 5 } IP $\leq \mathbf{0 , 6 5}$ & $\%$ & $\mathbf{n}$ & $\%$ \\
IP $\leq 0,65$ & 4 & $22,2 \%$ & 22 & $10,3 \%$ \\
IP $>0,65$ & 14 & $77,8 \%$ & 192 & $89,7 \%$ \\
IP $\leq \mathbf{0 , 7 5}$ & & & & \\
IP $\leq 0,75$ & 9 & $50 \%$ & 44 & $20,6 \%$ \\
IP $>0,75$ & 9 & $50 \%$ & 170 & $79,4 \%$ \\
Total & $\mathbf{1 8}$ & $\mathbf{1 0 0 \%}$ & $\mathbf{2 1 4}$ & $\mathbf{1 0 0} \%$ \\
\hline
\end{tabular}




\section{DISCUSIÓN}

El aumento de la resistencia en las uterinas está mayormente relacionado con restricción del crecimiento intrauterino y preeclampsia ${ }^{21-24}$, pero no está demostrado que una baja resistencia del flujo sanguíneo uterino esté directamente relacionada con la presencia de macrosomía fetal. Aunque el argumento tiene lógica, resulta por demás extraño que no se haya reparado en este detalle, el cual tiene argumentos fisiológicos y fisiopatológicos sólidos que por un raciocinio lógico lo demuestran, como es por ejemplo que a mayor volumen sanguíneo mayor peso fetal. Los hallazgos del presente estudio que muestran que la media de los índices de pulsatilidad de las arterias uterinas antes de las 30 semanas tienen una tendencia hacia los valores bajos, apoyarían lo anteriormente expuesto. Por ello, se recomienda realizar estudios futuros de mayor rigor metodológico que permitan demostrar mejor la asociación entre la media del IP de las uterinas y la macrosomía fetal.

Una de las principales contribuciones del presente estudio sería ayudar a disminuir la obesidad que es un problema de salud mundial, porque la prevención de la obesidad debe hacerse desde la etapa fetal, debido a que se ha demostrado en el ser humano que la hiperplasia de los adipocitos se inicia en la etapa fetal y continúa durante los primeros años de vida hasta los cinco años de edad; y de allí solo ocurre hipertrofia de los adipocitos ya formados ya no existe hiperplasia. Por lo tanto, el objetivo de la prevención sería evitar la hiperplasia de los adipocitos y no tanto su hipertrofia, por lo que es mejor combatir la obesidad desde la vida feto antes que de en la etapa adulta.

En este contexto, se podría diferenciar a las pacientes con valores de la media del IP de las arterias uterinas $\leq$ 0,75 para que ingresen a un plan de régimen nutricional adecuado para evitar que sus bebés presenten macrosomía fetal.

Se recomienda incluir en futuras investigaciones otras variables que pueden ser confusoras como el nivel socioeconómico y el nivel educativo. Asimismo, se hace necesario realizar investigaciones similares en los diferentes medios socioculturales del país.

Se concluye que un valor de la media del índice de pulsatilidad de las arterias uterinas $\leq 0,75$ se asoció con macrosomía al nacer.

\section{REFERENCIAS BIBLIOGRÁFICAS}

1. Limay A. La Ganancia de peso materno en el tercer trimestre influye más en la macrosomía neonatal independientemente del peso materno pre gestacional. "Tesis Maestría Salud Publica UNMSM”. Perú 2005.

2. Ticona MR. Curva de referencia peruana del peso al nacimiento para la edad gestacional y su aplicación para identificar neonatos de alto riesgo . Revista de Salud Pública. Perú 2007.

3. Larrabure G, Córdova D, Espinoza I. To establish the birthweigth and their relationship with maternal nutritional state in the Maternal Perinatal Institute of Lima-Perú.

4. Goldenberg RL, Humphrey JL. Hale CB, et al. Neonatal deaths in alabama, 1970-1980: An analysis of birth weightand race-specific neonatal mortality rates. Am J Obstet Gynecol. 1983;145:545-555

5. Kloosterman GJ. On intrauterina growth: The significance of prenatal care. J.Gynecol Obstet. 1970;8:895

6. Ounsted M. Ounsted C. On fetal growth rate: Its variations and their consequences. In: Clinics in Development Medicine (46). Spastics International Medical Publications. Philadelphia, PA: Lippincott, 1974

7. Lubchenco LO, Hansman Ch, Dressler M, Boyd E. Intrauterine growth as estimated from liveborn birth-weigt data at 24 to 42 week of gestation. Pediatrics, 1.963, 32: 793-800

8. Fescina RH, y Schwarcz R. Crecimiento intrauterino. La mujer gestante. En Crecimiento y Desarrollo, editores Cuminsky M, Moreno E.M, y Suárez EN, OPS, Publicación Científica N510. 1988, Washington DC. Pag 71-89.

9. Fescina RH, Schwarcz R, DíazAG. Vigilancia del crecimiento fetal. Publicación Científica CLAP N¹261. Abril de 1996. Pag 9-10.

10. Brenner WE, Edelman DA, Hendricks ChH. A standard of fetal growth for the United States of America. Am J Obstet Gynecol 1976; 126: 555-564.

11. Ticona M, Huanco D. Macrosomía Fetal en el Perú Prevalencia, Factores de Riesgo y Resultados Perinatales. Rev Ciencia y Desarrollo- Perú 2005.

12. Veille JC, Figueroa JP, Mueller-Heubach E. Validation of noninvasive fetal renal artery flow measurement by pulsed Doppler in the lamb. American Journal of Obstetrics and Gynecology 1992; 167(6):1663-1667

13. Reuwer $A Q$, Reuwer $P J$, van der Post JA, Cramer MJ, Kastelein JJ. Año Human Reprod.1984 Nov; 18(4):199-205.

14. Thompson R. Trudinger B. Doppler waveform pulsatility index and resistance, pressure and flow in the umbilical placental circulation: An investigation using a mathematical model. Volume 16, Issue 5, 1990, Pages 449-458.

15. Doppler ultrasound waveform indices: $A / B$ ratio, pulsatility index and Pourcelot ratio.Trudinger B Thompson R. Colleen M. C. Volume 95, Issue 6, pages 581-588. June 1988.

16. Meta-análisis Base de Datos Oxford

17. Neilson JP, Alfirevic Z. Ecografía Doppler para la evaluación fetal en embarazos de alto riesgo (Revisión Cochrane traducida). En: La Biblioteca Cochrane Plus, 2008 Número 4. Oxford: Update Software Ltd. Disponible en: http://www. update-software.com.

18. Trudinger BJ, Cook CM, Giles W, et al. Fetal umbilical artery waveforms and subsequent neonatal outcome. $\mathrm{Br} \mathrm{J}$ ObstetGynaecol 1991; 98:378-84

19. McCowan LM, Erskine LA, Ritchie K. Umbilical artery Doppler blood flow studies in the preterm, small for gestational age fetus. Am J Obstet Gynecol 1987; 156(3):655-9.

20. Vilcas L. Incidencia y factores de riesgo de macrosomía fetal en el hospital II Suárez Angamos. Tesis para optar el título de especialista en Pediatría. UNMSM - 2007. LimaPerú.

21. Bracero L, Benck D, Kirshenbaum N. Doppler Velocimetry Wveforms and the Placental disease. Am J Obstet Gynecol 1989; 161:388. 
22. Fox H. Macroscopic abnormalities of the placenta. Pathology of the Placenta, Fox H (ed). W.B. Saunders: London, UK, $1997 ; 102-150$

23. Khong T, De Wolf F, Robertson WB, Brosens I. Inadequate maternal vascular response to placentation in pregnancies complicated by pre-eclampsia and small. $\mathrm{Br} \mathrm{J}$ Obstet Gynaecol 1986; 93: 1049-1059.

24. Peeters LL, Sheldon RE, Jones MD, Makowski EL, Meschia G. Blood flow to fetal organs. Am J ObstetGynecol 1979; 135:637-46.

25. Wladimiroff J, Tonge H, Stewart P. Doppler ultrasound assess- ment of cerebral blood flow in the human fetus. $\mathrm{Br}$. J Obstet Gynaecol 1986; 93:471-5.
26. Doppler JC. Ueber das farbigeLicht der Doppelsterne und einigeandere Gestirne des Himmels. Abhandlung der Königl. Böhmischen Gesellschaft der Wissenschaft Sers.2 1843; 465-82.

27. Gosling RG, King DH. Arterial assessment using Dopplershift ultrasound. Pro R Soc Med 1974; 67:447-449.

28. J Albornoz, H Salinas, A Reyes. Morbilidad fetal asociada al parto en macrosómicos: análisis de 3981 nacimientos. Rev. Chil Obstet Ginecol 2005; 70(4): 218-224.

29. Cutié M, Figueroa M, Segura A. Macrosomía fetal. Su comportamiento en el último quinquenio. Hospital Militar Central "Dr. Luis Díaz Soto" Rev Cubana Obstet Ginecol 2002; 28(1):34-41.

\section{ORCID iDs}

Antonio Limay Rios

Elías Alexis Valladares Gutiérrez

Erasmo Huertas Tacchino

Walter Castillo Urquiaga (7i) https://orcid.org/0000-0001-6012-3705

(i) $h$ ttps://orcid.org/0000-0003-2789-4764

(i) https://orcid.org/0000-0002-9851-8419

(di) https://orcid.org/0000-0002-1054-7398 